\title{
Highly Anisotropic Vorticity Aligned Structures in a Shear Thickening Attractive Colloidal System
}

\author{
Chinedum O. Osuji \\ Department of Chemical Engineering \\ Yale University, New Haven CT 06511 \\ E-mail: chinedum.osuji@yale.edu
}

\author{
David A. Weitz \\ School of Engineering and Applied Sciences \\ Harvard University, Cambridge MA 02138. \\ Email:weitz@seas.harvard.edu
}

November 16, 2018

Vorticity aligned cylindrical flocs of carbon black particles are formed in steady flow at low shear rates, and strikingly, appear as transient structures in the flow response of gels produced by the quenching of high rate shear thickening flows.

Structure formation in response to flow is common in soft materials and complex fluids and is dependent on the nature of the cohesive forces in the system, time scales for diffusive motions and hydrodynamic interactions. For Brownian systems with hard sphere or repulsive interactions, distortion of the microstructure due to an imposed shear rate occurs when the timescale for flow is smaller than that for diffusion. The relative strength of flow forces compared to thermal or diffusive forces can be gauged by the dimensionless Péclet number

$$
P e=\frac{\eta \dot{\gamma} a^{3}}{k_{B} T}
$$

For $P e \ll 1$ the system displays isotropic arrangements of particles, but for $P e>1$ the timescale associated with flow dominates that for diffusive relaxation. Under these conditions, the system becomes anisotropic due to separation of particles along the elongational axis and aggregation along the compressional axis [1]. String formation by alignment along the flow direction is typical in dilute systems at high shear rates and further structuring may take place by periodic arrangement of these strings in the vorticity-gradient plane. In more concentrated suspensions, hydrodynamic interactions lead to the formation of hydro-clusters and a shear thickening or jamming transition [2, 3]. In systems with constitutive instabilities due to a negative slope in the stress-strain curve, co-existence between different deformation states under a single applied stress gives rise to shear banding along the velocity gradient direction [4]. For attractive systems, structure formation as discussed above is also found. Additionally, the alignment of particles or constituents along the vorticity axis has been reported and has emerged as a somewhat general phenomenon [5, 6]. Shear thickening though, is not generally observed in colloidal systems with sufficiently strong attractive interactions to form flocculated gels and is presumed not to occur [7]. It has been reported in a handful of systems to date [8, 9, 10], although the complex composition of the suspensions studied in some cases makes it difficult to unambiguously study the mechanism.

We have studied the steady state flow behavior of dilute, simple hydrocarbon dispersions of carbon black particles and, surprisingly, observe shear thickening above a composition dependent critical flow rate, $\dot{\gamma}_{c} \approx 10^{2}-10^{3} \mathrm{~s}^{-1}$. The shear modulus of gels formed by pre-shearing above the critical shear thickening rate displays an interesting power law dependence on the stress applied during the pre-shear. This is well accounted for by the dependence of the cluster size on the applied shear stress, and the resulting increase in cluster number density [11]. Subsequent deformation of these shear thickened gels at low shear rates produces highly oriented vorticity aligned flocs that gradually break down into small isotropic clusters over timescales of $\approx 300$ seconds.

We use tetradecane (Aldrich Chemical Co.) dispersions of 2 to 8 wt. $\%$ of $0.5 \mu \mathrm{m}$ carbon black particles (Cabot Vulcan XC72R) with a fractal dimension $d_{f}=2.2$. Rheological data reported here were recorded using an ARG2 (TA Instruments) rheometer in strain control, although separate experiments were also run for verification purposes on a pure-strain controlled ARES-LS1 instrument. A variety of geometries, standard and roughened, were used to check for and to mitigate the impact of possible wall slip. Although from our measurements it appears that wall slip is not significant, its preva- 
lence in other studies on "sticky" colloidal gels makes the interpretation of data at low shear rates troublesome [12]. A more detailed study of the low shear rate regime is underway. Optical observations of microstructures under shear were made using a Bohlin CS rheometer with a transparent base-plate through which images were recorded (by reflection off the rheometer top tool) using a CCD camera.

The flow curves for samples in steady shear display thixotropy, as is typical for flocculating systems. Steady state flow curves of the shear rate dependent stress display a composition-dependent, continuous shear thickening transition at high shear rates, $\dot{\gamma}_{c} \approx 10^{2}-10^{3}$, with distinct increases in the stress and thus the viscosity of the dispersions with increasing shear rate, Figure 2 . From optical observations, Figure 1, at low shear rates $\dot{\gamma} \lesssim 1 s^{-1}$, the system is composed of large pieces of the fractured gel which gradually break into smaller pieces with increasing shear rate. Around $\dot{\gamma} \approx 10^{0}-10^{1} s^{-1}$, we observe a slight, if irregular increase in viscosity with increasing shear rate. Coincident with flow in this region, we observe the aggregation of clusters along the vorticity axis, and the formation of elongated flocs which roll with the shear flow. Such flocs are also observed in long observations overnight at constant shear rate, indicating that, within the experimental time frame, they represent the steady state response of the system to flow in this range of shear rates. Continued increase of the shear rate results eventually in dissolution of the cylindrical structures into isotropic clusters. These clusters densify until $\dot{\gamma}_{c}$ beyond which there is a transition to the shear thickening flow as breakage of the aggregates is observed, consistent with an increase in the effective volume fraction of particles in the dispersion.

Previous studies have implicated negative first normal stress differences, $N_{1}=\sigma_{11}-\sigma_{22}$, in the formation of vorticity aligned structures in flocculating systems [5, 6]. Normal stresses arise due to elasticity in the flowing suspension with elongational stiffness and bending rigidity both contributing [13, 14, 15. In our system, we are unable to identify any systematic changes in normal stress at low shear rates. It may simply be that the stresses are too small to be accurately measured in our rheometer. In the shear thickening regime however, samples develop large negative normal stresses, on the order of $N_{1} \approx 2-10 . \sigma_{\text {shear }}$, depending on composition, due to the large inertia developed at these high shear rates. Abrupt cessation of shear in this regime produces a quench into a shear-thickened gel state where, remarkably, both shear stresses and normal stresses were found to persist for

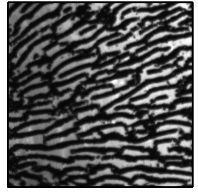

a

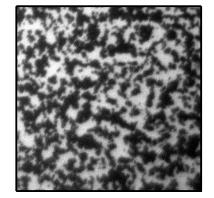

b

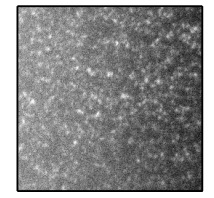

C
Figure 1: Microstructure under shear in parallel plate geometry with a gap size of $100 \mu \mathrm{m}$ for $3 \mathrm{wt} . \%$ sample. (a) $\dot{\gamma}=6.67 \mathrm{~s}^{-1} 40 \mathrm{~mm}$ field of view showing cylindrical flocs aligned along the vorticity direction. (b) $1.5 \mathrm{~mm}$ field of view, $\dot{\gamma}=133 \mathrm{~s}^{-1}$ during shear (c) $1.5 \mathrm{~mm}$ field of view, $\dot{\gamma}=1330 \mathrm{~s}^{-1}$ during shear. To obtain sufficient light transmission through the optically dense sample in (c), the illumination was set several times higher than that used for (b). Binning of pixels on the CCD was used to decrease the required exposure time and so the resolution of image (c) is half that of (b).

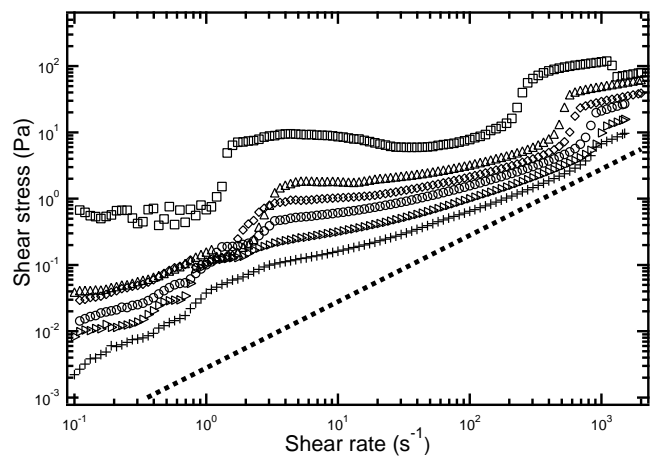

Figure 2: Steady state flow curve of carbon black dispersions from 2 to 8 wt. \%.+ : $2 \%, \triangleright: 3 \%, \circ: 4 \%, \diamond: 5 \%$, $\triangle: 6 \%, \square: 8 \%$ The dotted-line shows the stress due to the background viscosity of the solvent.

long times ( $>1$ hour) under quiescent conditions and to dissipate rapidly on application of shear flow. We have studied the dynamics of the relaxation of the internal shear stresses and find that they relax with a power law dependence $\sigma_{i} \sim t^{-0.1}$ over timescales as long as $10^{3}-10^{4} \mathrm{~s}$ [11]. Accurate measurement of the modest normal stresses in dilute suspension rheology is made difficult by the lack of better than $\approx 10-20 \mathrm{~Pa}$ resolution available on most commercial rheometers, which rely on a strain gauge style mechanism that is usually not stabilized against temperature fluctuation. The presence of a large inertial contribution [16], $N_{1}^{\text {inertial }}=-3 / 20 \rho \omega^{2} R^{2}$ to our signal further complicates this in our case, but from more concentrated samples, $\phi>4$ wt.\%, we could 
reliably extract the sample contribution to the normal stress, Figure 3 . Correction of the data for inertial contributions shows that a positive normal stress difference is developed at the onset of shear thickening, consistent with the idea that shear thickening, as indicated from optical observation, is due to cluster breakage and an increase in the effective volume fraction of the system. The negative normal stress observed at rest relative to the flowing state appears to originate during the rapid quench from the tendency of the particles to collapse into dense clusters on cessation of shear. Accurate measurements of the normal stress transients as well as the normal stress dynamics in the quiescent state are the subjects of continuing work.

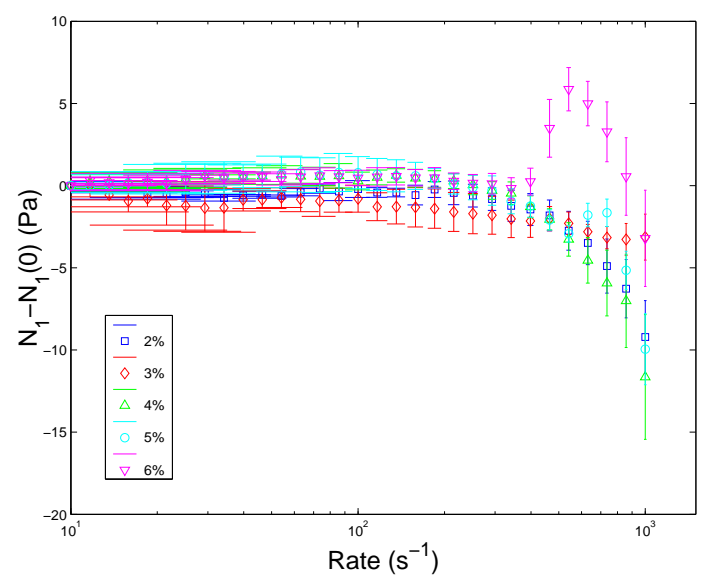

Figure 3: First normal stress difference (relative to starting value, $N_{1}(0)$ )for various compositions as a function of shear rate. Data are averaged over 4 runs. Error bars correspond to one standard deviation.

The deformation of quenched shear thickened gels by steady flow $\left(0.1 s^{-1} \lesssim \dot{\gamma} \lesssim 10 s^{-1}\right)$ or large amplitude oscillatory shear $(\omega=1 \mathrm{rad} / \mathrm{s}, \gamma \geqslant 50 \%)$ results in the rapid emergence of highly anisotropic vorticity aligned structures. The transition from shear thickened 3 -dimensional gel to an assembly of discrete cylindrical flocs in rolling flow for a sample of 2 wt.\% carbon black, using a parallel plate geometry, is shown in Figure 4 (see supplemental data for video). In contrast to the structures observed in the steady state response at low shear rates, these transient flocs were much more sharply defined and with aspect ratios exceeding $10^{2}$, spanned macroscopic portions of the shear cell.

Fourier transformations of the images provide a means of following the formation and alignment of the vorticity aligned flocs. We inspect a small section of the sample, 3 $\mathrm{mm}$ on a side and $2.5 \mathrm{~mm}$ from the tool edge, to limit the

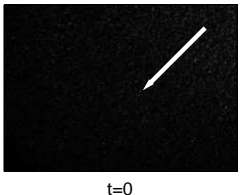

$\mathrm{t}=0$

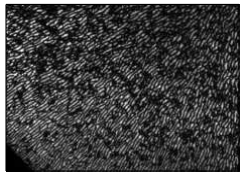

3s

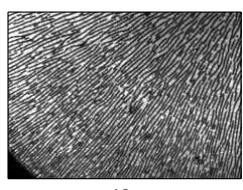

$12 \mathrm{~s}$

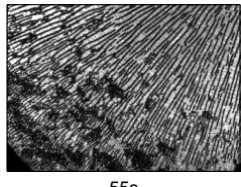

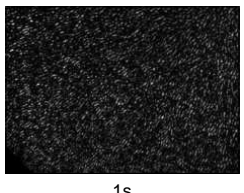

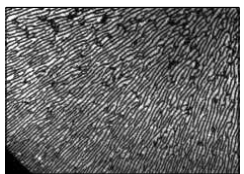

$6 s$

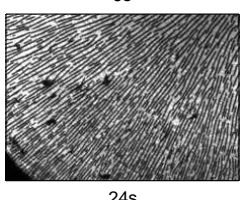

$24 \mathrm{~s}$

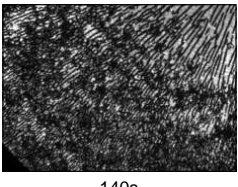

140 s
Figure 4: Carbon black dispersions, $\phi=2 w t . \%$, shear thickened by strong flow in the secondary thickening regime. Samples are quenched to zero-shear rate and then subsequently deformed, starting at $t=0$ with a shear rate of $\dot{\gamma}=10 \mathrm{~s}^{-1}$. Parallel-plate geometry with gap-size $d=50 \mu \mathrm{m}$. Field of view is $1.35 \mathrm{~cm}$ in width. Vorticity direction is along the bottom-left to top-right diagonal as given by the arrow in the first image.

distortion due to the circular geometry. We are unable to resolve the rapid changes in the characteristic length scale of the system due to the low optical resolution of the camera as well as the short timescale at which a stable structure emerges, Figure 5. We can, however, monitor the development of alignment in the system. We extract the azimuthal intensity dependence of the FFTs at the spatial frequency corresponding to the periodicity of the flocs and model them with a Gaussian. As shown in Figure 5, alignment is rapid and peaks around 20s, $\gamma=200$, after which it quickly decreases as the flocs break up.

Using the parallel-plate geometry, we examined the dependence of the floc geometry on physical confinement. The width of the rolling log-like structures measured at a distance of $2 \mathrm{~mm}$ from the tool edge, $w_{l o g}$, was moderately larger than the gap size, $d$, and varied in direct proportion with the gap, Figure 6. The accuracy in determining the log width is limited by the $20 \mu \mathrm{m}$ per pixel resolution at which data was taken, but in general, 

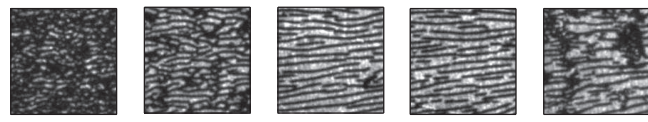

(a)
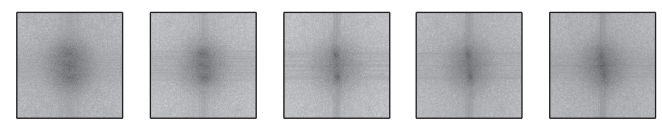

(b)

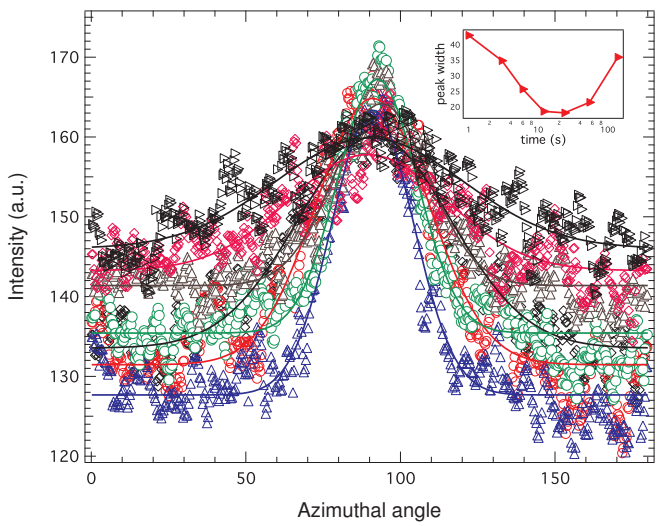

Figure 5: (a) $3 \mathrm{~mm}$ sections of optical micrographs over time. Gap $=50 \mu \mathrm{m}$. From left to right: $t=1,3,12,24$ and $55 \mathrm{~s}$ (b) Azimuthal spread of intensity from FFTs of images in (a). Solid lines are Gaussian fits to the data. Inset: Peak widths vs. log time

$w_{l o g} \approx 1.4 d$. At gaps larger than $750 \mu m$, the structures could no longer be clearly discerned. From the images, it can be seen that smaller gaps promote sharper interfaces between the structures and the surrounding fluid. The width of the structures increases slightly with decreasing shear rate in moving from the outside of the parallel-plates towards the center. Increasing gap size results in greater areal coverage of the parallel-plates as the periodicity of the logs does not increase commensurate with their width. This implies that the flocs become less dense internally with increasing thickness. At long times, the dissolution of the structure proceeds from the outside edge of the tool, where the shear rate is highest. In observations employing a low angle cone $\left(1^{\circ}\right)$ where the shear rate is now constant across the tool, dissolution proceeds from the outside edge where the gap is largest, underscoring the importance of confinement in stabilizing the flocs.

The frequency and strain dependence of the elastic modulus of the log-like flocs differ from those of the shear thickened gel. Here, the vorticity-aligned structures were produced by stopping shear flow at $t=20 \mathrm{~s}$ in a $40 \mathrm{~mm}, 1^{\circ}$ cone-plate cell with an edge gap of $350 \mu \mathrm{m}$. Both the gel and the aligned structures show strong elas-

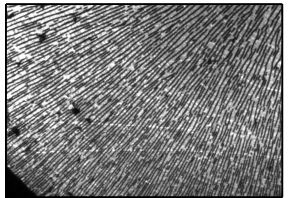

a

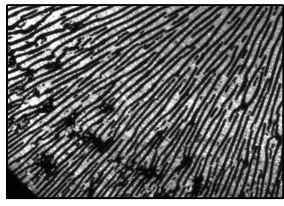

C

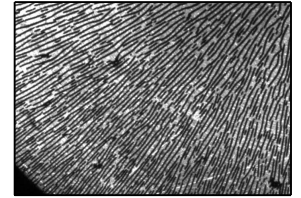

b

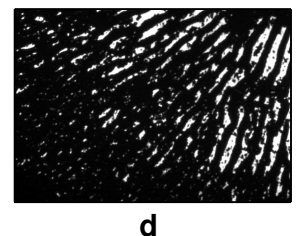

d
Figure 6: Microstructure of 2 wt. \% carbon black dispersions at $\approx 25 s, \dot{\gamma}=10 s^{-1}$ after shear thickening flow in $40 \mathrm{~mm}$ plate-plate geometry with various gaps, $d$. Field of view is $1.35 \mathrm{~cm}$ in width. (a) $d=25 \mu \mathrm{m}$ (b) $d=50 \mu \mathrm{m}$ (c) $d=100 \mu \mathrm{m}(\mathrm{d}) d=250 \mu \mathrm{m}$

tic responses, with nearly frequency-independent moduli. The yield strain, however, is markedly lower for the structures than for the gel, Figure 7 a. Yielding of the vorticity-aligned structures proceeds without the pronounced upturn in the loss modulus, $G^{\prime \prime}$ that typically signifies yielding in the gel, and the crossover between $G^{\prime}$ and $G^{\prime \prime}$ occurs at higher strains than is typical for the gel. The existence of a yield strain implies that displacement and deformation in the system is not accommodated solely by the rolling motion of the flocs.

Vorticity aligned structures have been observed in a variety of complex fluids such as thixotropic clay gels [17, nanotube suspensions [6] and attractive emulsion droplets [5], associated in each case with negative normal stress differences. In our system, the carbon black dispersions appear to display small negative normal stresses over all shear rates before increasing at the shear thickening transition and decreasing again beyond the peak of the transition. No distinct transition in normal stress was observed under steady flow at low shear rates, though this is subject to the difficulty involved in accurate measurements of small changes in these stresses due to instrument resolution. Measuring the absolute value of the normal stress is further complicated by the suction of the tool surface by the sample, i.e. in such systems it is not clear what constitutes absolute zero. Rather, significance can only be ascribed to differences in the normal force signal with respect to some initial condition.

The rapid formation and the high degree of coherence 
(a)

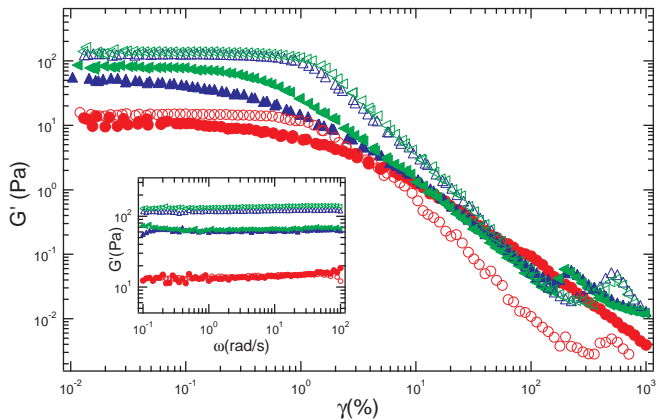

(b)

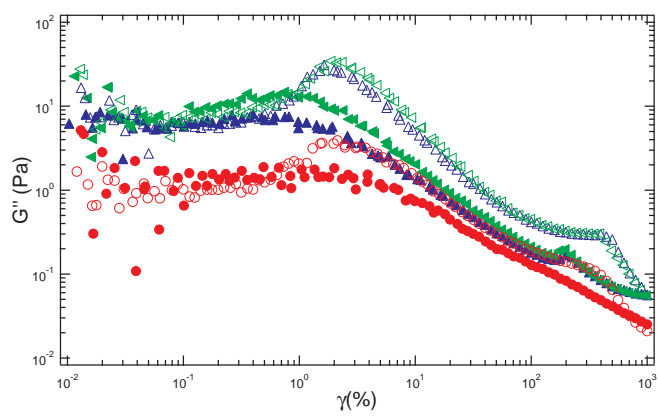

Figure 7: Strain dependent moduli of shear thickened gels (open symbols) and log-like flocs (filled symbols) for carbon black dispersions of various compositions. Strain data taken at $\omega=1 \mathrm{rad} / \mathrm{s}$. and frequency data at $\gamma=$ 0.05\%. ०: 2 wt.\%; $\triangle: 3$ wt.\%; $: 4$ wt.\%.(a) Storage modulus. Inset: Frequency dependent data. (b) Loss modulus.

of the structures formed in the transient flow response of the gel suggest strongly that relaxation of quenched normal stresses play a significant role. The dissipation of the normal stress with continued flow precludes long term stability of the structures and as a result they exist only transiently. Our results are similar to observations of an elastic instability associated with flow-induced clustering in semi-dilute non-Brownian nanotubes [18]. Here, the transient rheological response of a flowing suspension of nanotubes subjected to a shear-rate quench is underlined by the formation of vorticityaligned aggregates. The existence of these aggregates can be parameterized by confinement and shear rate. In our system, however, we also observe these structures as a response to steady flow at low shear rates. By stopping the flow of shear-thickened systems during the transient response, we have been able to access measurements of the frequency and strain dependence of the shear modulus of these structures. The transition from a three-dimensionally connected network to a two-dimensional system of highly anisotropic structures and the dissolution of these structures thereafter shows interesting features. Locally, one can discern changes in topology as the flocs make and break connections with neighboring structures, and track changes in the order parameter as shear finally dissolves the system into non-aligned clusters. The instrument used in this study did not permit the consistent application of arbitrarily small deformations. As a result, we were unable to conduct measurements at the low strain rates that would have permitted better resolution of the dynamics of structure formation and dissolution in our samples. Future work will benefit from a more capable instrument that also permits measurements of normal forces and will pursue more detailed studies of the effect of confinement and composition on the mechanics of these structures.

The authors gratefully acknowledge C. Kim and H. Wyss for very helpful discussions, and the Infineum Corporation for funding.

\section{References}

[1] J. Vermant and M. J. Solomon, Journal of PhysicsCondensed Matter, 2005, 17(4), R187-216.

[2] A. A. Catherall, J. R. Melrose, and R. C. Ball, Journal of Rheology, 2000, 44(1), 1-25.

[3] M. K. Chow and C. F. Zukoski, Journal of Rheology, 1995, 39(1), 15-32.

[4] J. Vermant, Current Opinion in Colloid $\&$ Interface Science, 2001, 6(5-6), 489.

[5] A. Montesi, A. A. Pena, and M. Pasquali, Physical Review Letters, 2004, 92, 058303.

[6] E. K. Hobbie, S. Lin-Gibson, H. Wang, J. A. Pathak, and H. Kim, Physical Review E, 2004, 69, 061503.

[7] V. Gopalakrishnan and C. F. Zukoski, Journal of Rheology, 2004, 48(6), 1321-1344.

[8] A. Potanin, Journal of Rheology, 2004, 48, 1279.

[9] H. Kanai and T. Amari, Rheologica Acta, 1995, 34(3), 303-310.

[10] M. Kawaguchi, M. Okuno, and T. Kato, Langmuir, 2001, 17(20), 6041-6044.

[11] C. O. Osuji, C. Kim, and D. A. Weitz, Submitted to Phys. Rev. E. 
[12] H. Walls, S. Caines, A. Sanchez, and S. Khan, Journal of Rheology, 2003, 47, 847.

[13] K. G. Kang, M. P. Lettinga, Z. Dogic, and J. K. G. Dhont, Physical Review E, 2006, 74, 026307.

[14] S. Kharchenko, J. Douglas, J. Obrzut, E. Grulke, and K. Migler, Nature Materials, 2004, 8, 564-568.

[15] L. Becker and M. Shelley, Physical Review Letters, 2001, 87(19), 198301.

[16] W. Kulicke, G. Kiss, and R. Porter, Rheologica Acta, 1977, 16(5), 568-572.

[17] F. Pignon, A. Magnin, and J. M. Piau, Physical Review Letters, 1997, 79, 4689.

[18] S. Lin-Gibson, J. A. Pathak, E. A. Grulke, H. Wang, and E. K. Hobbie, Physical Review Letters, 2004, 92, 048302. 